\title{
Spectrum of Organisms and Correlation of Gram-Smear and Quantitative Cultures of Endotracheal Aspirates and Broncho Alveolar Lavage Fluid with Clinical Criteria for the Diagnosis of Ventilator Associated Pneumonia
}

\author{
Sumana Prudhivi ${ }^{1 *}$, Uma Sekhar ${ }^{2}$, Anupama Jyoti Kindo ${ }^{2}$ and Mallika ${ }^{2}$ \\ ${ }^{1}$ Department of Microbiology, NRI Medical College, Chinakakani - 522503, Mangalagiri \\ Mandal, Guntur District, Andhra Pradesh, India \\ ${ }^{2}$ Department of Microbiology, Sri Ramachandra University, No: 1, Ramachandra Nagar,Porur, \\ Chennai, Tamilnadu -600116, India \\ *Corresponding author
}

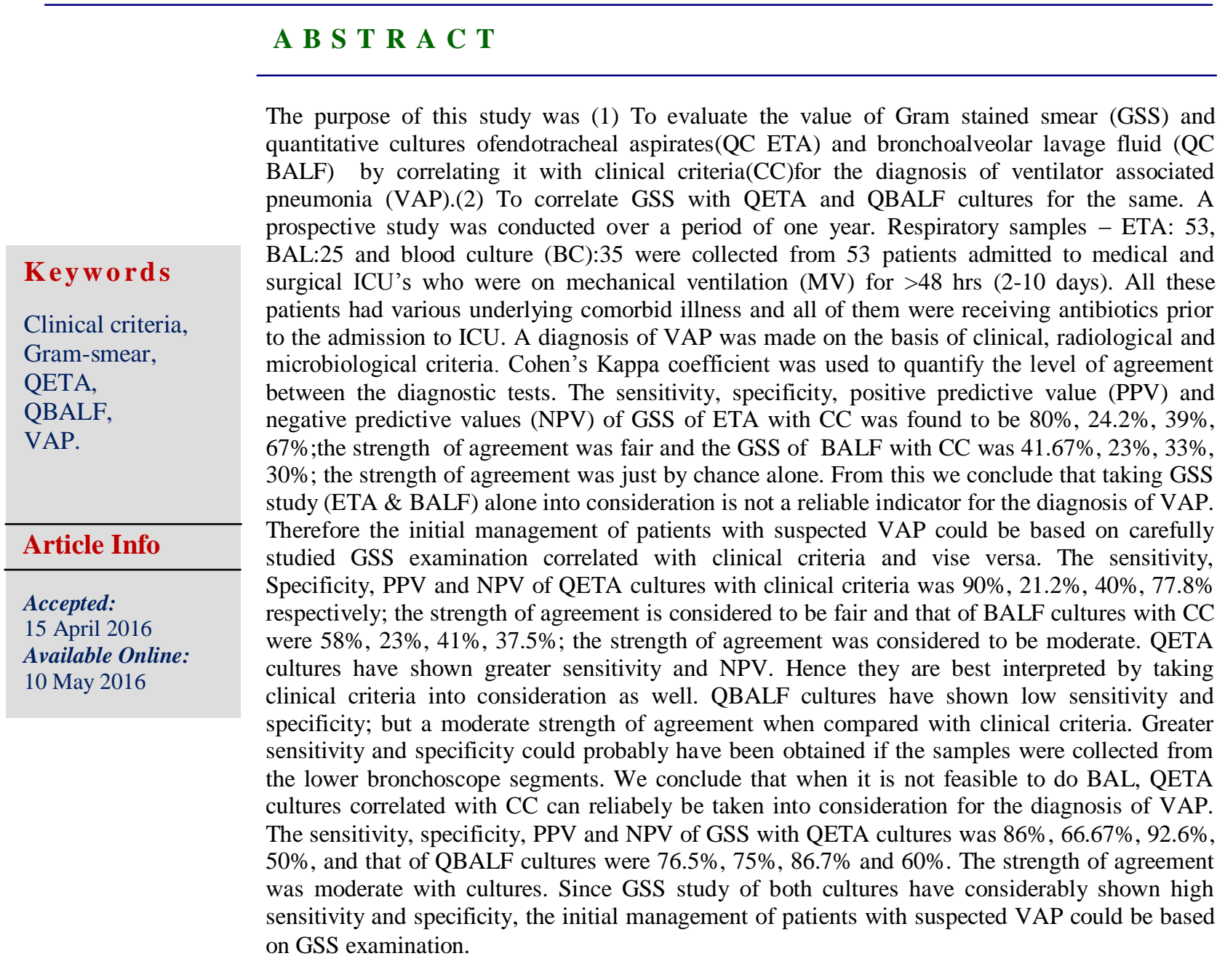




\section{Introduction}

VAP is a complication that occurs in appropriately $9-27 \%$ of ICU patients more than $48 \mathrm{hrs}$ after endotracheal intubation and mechanical ventilation (American Thoracic Society, 2005; Craven et al., (2005)). The diagnosis of VAP is challenging because the clinical and radiological findings lack sensitivity and specificity. Priorinstitution of antibiotic therapy and upper airway colonization further complicates the microbial analysis (Mozzamil Shafi et al., (2015)). Cultures of lower respiratory tract samples, such as protected specimen brush, Bronchoalveolar lavage fluid or Open lung biopsy, have demonstrated high predictive value for the identification of VAP causative microorganisms and are frequently considered superior to endotracheal aspirates (Medford et al., (2009); Baselski et al., (1993). In contrast ETA samples are more readily obtainable from mechanically ventilated (MV) patients and is frequently a component of microbiogical surveillance (Brusselaers et al., (2013)). Since colonization precedes infection, bacteria identified by cultures of ETA'S may indeed be the causative microorganisms. once VAP develops (Bontenet et al., (1996) ETA nonquantitative cultures nevertheless lack a validated threshold for positivity and, consequently they may not distinguish colonization from infection and may be non indicative for VAP (Medford et al., (2009), Brusselaers et al., (2013), Berton et al.,). Only a few guidelines discourage the use of ETA's for VAP diagnosis (Masterton et al., 2008), the majority of VAP guidelines recommend the use of either ETA or BALF analysis to diagnose VAP (American Thoracic Society 2005, Raoof and Bauman 2014).

Diagnostic and treatment strategies in ICU patients with VAP remain controversial.
Clinical diagnosis of VAP is not an easy task for ICU physicians. The lower respiratory tract specimens obtained by various techniques (non-invasive \& invasive) provide an insight into the etiology (American Thoracic Society 2005, Camargo et al., (2004) and act as an adjuvent to clinical diagnosis in the management of VAP. Keeping in view of this rising importance of VAP in ICU's, this study compared (1) Gram-smear and QC's of ETA and BALF with clinical criteria in patients with suspected VAP. (2) Gram-smear with quantitative cultures of ETA and BALF. (3) An attempt was also made to compare the results of QETA, QBALF and Blood cultures in permissible cases.

\section{Materials and Methods}

A prospective study was conducted over a period of one year. Respiratory samples ETA: 53, BAL: 25 and blood samples: 35 were collected randomly from 53 patients admitted to Medical and Surgical ICU's, who were on mechanical ventilation for greater than 48 hours. All these patients had various underlying co-morbid illness and all of them were receiving antibiotics prior to the admission to ICU.A diagnosis of VAP was made on the basis of clinical, radiological and microbiological criteria. (Grant et al., (2001), Mauricio et al., (2000), el-Ebairy et al., (1993), Inglis et al., (1989), Mozzamil et al., (2015)).

\section{Clinical Criteria (Mauricio et al., (2000))}

1. Fever $\geq 38^{\circ} \mathrm{c}$ or hypothermia $\leq 35^{\circ} \mathrm{c}$.

2. Purulent respiratory secretions.

3. Leucocytosis > $>12,000 / \mathrm{mm} 3$ or Leucopenia $<4000 / \mathrm{mm} 3$.

4. Selection of patients on ventilator for greater than 48 hours. 


\section{Radiological Criteria}

Presence of new and/or progressive chest radiographic infiltrates appearing after 48 hours of mechanical ventilation. The combination of any two clinical features with an abnormal chest radiograph is taken as gold standard.( Mauricio et al., (2000))

Microbiological Criteria: (el-Ebairy et al., (1993), Mozzamil et al., (2015))

Gram-stain of respiratory secretions (ETA\&BAL) showing: $\geq 25$ pus cells/10x,

$\leq 10$ squamous epithelial cells/10x, Intracellular organisms (ICO) -4 ICO in 200 cells $=105 \mathrm{cfu} / \mathrm{ml}$.

QETA cultures showing a significant growth of $>10^{6} \mathrm{cfu} / \mathrm{ml}$ and QBAL cultures showing a significant growth of $>10^{4} \mathrm{cfu} / \mathrm{ml}$. (Steven et al., (1997), el-Ebairy et al., (1993))

\section{Specimen Collection}

ETA, BAL and blood samples collected from mechanically ventilated patients were processed within half an hour of arrival to the microbiology laboratory .ETA's collected by trained ICU nurses using Ramson's mucus extractor GS-5018W without filter. Bronchial wash was performed by the chest physician using flexible Olumpus Fiber Optic Bronchoscopy (FOB), type 2E under the guidence of pulseoxymetry and visualisation. The procedure is repeated 3 or 4 times and the secretions collected into 3 sterile test-tubes.

Laboratory processing of specimens: Gramstaining of ETA and BAL samples were performed using undiluted samples. Smears were examined for the presence of (1)Quality of sample (2)Pus cells and epithelial cells (3)Potential pathogen and intracellular organisms (ICO) under low power and oil immersion.

\section{ETA and BAL Cultures}

An equal volume of sterile $1 \% \mathrm{~N}$-acetyl-Lcysteine was added to the original respiratory secretions. The mixture of secretions and liquefying solution was incubated at room temperature for 10 minutes and vortexed for 2 minutes. Serial dilutions of 1:10, 1:100 and 1:1000 of each sample was prepared in sterile normal saline (Bartlet et al., (1996)). $100 \mathrm{ml}$ of each dilution is inoculated into the following agar media. $5 \%$ sheep blood agar (BA), chocolate agar (CHA) and MacConkey agar (MacA) All culture plates were incubated at $37^{\circ} \mathrm{C}$ aerobically with $10 \% \mathrm{CO}_{2}$. Cultures were evaluated for growth 24 and 48 hours later. Results were expressed as: Number of colonies $x$ dilution factor $x$ inoculation factor $=\mathrm{cfu} / \mathrm{ml}$. Recovery of potential pathogen showing a count of ETA $\geq 10^{6}$ $\mathrm{cfu} / \mathrm{ml}$ and $\mathrm{BALF} \geq 10^{4} \mathrm{cfu} / \mathrm{ml}$ was taken as the significant growth. Blood Samples were inoculated into blood culture bottles, incubated at 370c for 16-18 hours. Subculturing was done onto BA, CHA and Mac A plates at 24 hours, 48 hours and 72 hours for 1 week and plates were examined for growth. Bacterial isolates were identified by conventional standard technique (Colle et al., (2007))

\section{Statistics}

The sensitivity, specificity, positive and negative predictive values including 95\% confidence intervals, were calculated using the VAP diagnosis based on clinical criteria. Cohens kappa coefficient was used to quantify the level of agreement between the three tests. Graph pad soft ware was used for analysis. 


\section{Results and Discussion}

Total number of patients enrolled in the study were 53.Of which, 46 of them were males $(76.67 \%)$ and 14 were females $(23.3 \%)$. Of these maximum male patients were in the age group of 50-69 years and females in the age group of 60-69 years. The mean and standard deviation of age was $53.16 \pm 15.45$ and male to female ratio was 3.5:1 (41/12). Patients admitted to ICU had various underlying illnesses (Figure-1).

Of the 53 patients in whom ETA culture was done, 34 of them have shown growth. P.aeruginosa was the commonest organism isolated and no growth was seen in 19 patients. Out of 25 patients in whom BAL culture was done, 18 of them have shown growth. A.baumannii was the commonest organism isolated and no growth was seen in the remaining 7.Blood culture was done in 35 patients of whom 10 patients have shown growth and the commonest organism isolated was $\mathrm{S}$ aureus (Table-1).

Comparison of ETA Gram smear with clinical criteria. (Table 2, Fig 2): Among 53 patients $16(39 \%)$ were fitting into clinical criteria. Of the $12(22.6 \%)$ patients in whom Gram's smear was not suggestive of VAP, $8(67 \%)$ patients were not fitting into clinical criteria. Gram-stain sensitivity, specificity, PPV and NPV were $80 \%, 24.2 \%, 39 \%$ and $67 \%$.Cohen's Kappa coefficient $\mathrm{K}=0.035$, $95 \%$ Confidence interval $=-0.154$ to 0.225 . The strength of agreement is considered to be fair.

\section{Comparison of BAL Gram Smear with Clinical Criteria (Table-3, Fig-3)}

Among 25 patients in whom Gram- stain smear study was performed, 15(60\%) were suggestive of VAP of which 5(33\%) were fitting into clinical criteria of the $10(66.6 \%)$ in whom Gram smear was not suggestive of
VAP, $3(30 \%)$ patients were not fitting into clinical criteria. Gram's stain sensitivity, specificity, PPV and NPV are $41.67 \%, 23 \%$, $33 \%$, and $30 \%$. Cohen's Kappa coefficient $\mathrm{K}=-0.349,95 \%$ confidence interval is from -0.710 to 0.011 . The strength of agreement is just by chance alone.

\section{Evaluation of ETA Culture with Clinical Criteria (Table 4, Fig 4)}

Among 53 patients in whom culture was done, $44(77.78 \%)$ of them were showing significant growth of which $18(41 \%)$ were fitting into clinical criteria. Of the $9(19.6 \%)$ patients not showing significant growth, $7(78 \%)$ were not fitting into clinical criteria. Culture Sensitivity, Specificity, PPV and NPV's are 90\%, 21.2\%, 40\%, $77.78 \%$. Cohen's Kappa coefficient $\mathrm{K}=0.091$, 95\% Confidence interval is from -0.068 to 0.249 .The strength of agreement is considered to be fair.

\section{Evaluation of BAL Culture with Clinical Criteria (Table -5, Fig -5)}

Among 25 patients in whom culture was done $17(68 \%)$ of them have shown significant growth, of which $7(14 \%)$ were fitting into clinical criteria, of the $8(32 \%)$ patients in whom significant growth was not seen, $3(37.5 \%)$ were not fitting into clinical criteria.

Culture Sensitivity, Specificity, PPV, and NPV's are $58 \%, 23 \%, 41 \%, 37.5 \%$. Cohen's Kappa coefficient $\mathrm{K}=0.483, \quad 95 \%$ Confidence interval is from -0.132 to 0.834 .The strength of agreement is considered to be moderate.

Comparison of ETA Gram-stain with Culture (Table-6, Fig -6)

3 of the 53 patients in whom Gram's-stain smear study and culture was performed, $41(77.3 \%)$ of them were showing Gram's 
smear suggestive of VAP, of which $38(93 \%)$ were showing significant growth. Of the $12(22.6 \%)$ not suggestive of VAP, $6(50 \%)$ patients were also not showing significant growth. Gram-stain sensitivity, specificity, PPV and NPV's are 86\%, 66.67\%, 92.6\%, and 50\%.Cohen's Kappa coefficient $\mathrm{K}=0.468$, 95\% Confidence interval is from 0.175 to 0.762 . The strength of agreement is considered to be moderate.

\section{Comparison of BAL Gram-stain with Culture (Table-7, Fig -7)}

Of the 25 patients in whom Gram's-stain smear study and culture was performed, $15(60 \%)$ of them were showing Gram's smear suggestive of VAP, of which 13(87\%) were also showing significant growth. Of the 10(40\%) Gram's smear not suggestive of VAP, 6(60\%) patients were also not showing significant growth. Gram-stain Sensitivity, specificity, PPV and NPV are $76.5 \%, 75 \%, 86.7 \%$ and 60\%.Cohen's Kappa coefficient $\mathrm{K}=0.483, \quad 95 \%$ Confidence interval is from 0.132 to 0.834.The strength of agreement is considered to be moderate.

BAL and PSB are the most commonly used methods for diagnosing VAP although they require bronchoscopy. ETA is a simple and less costly technique than PSB or BAL. Criteria for definite and probable VAP has been developed and the definition is designed to eliminate pneumonia that is incubating or present at the time of intubation. The study was conducted on 53 mechanically ventilated patients of either sex admitted to ICU.

The mean age of patients is 53.16 with a standard deviation of 15.45 and maximum number of patients were between age group 50-69 years. This only reflects the age of patients getting admitted into ICU. Male to female ratio was 3.5:1 which reflects increased disease prevalence among males as well as custom of seeking admission to hospitals being more prevalent in males in our country.

Patients admitted to ICU had varying underlying illnesses. In this study, QETA cultures revealed high prevalence of MRSA and P.aeruginosa where as QBALF cultures have shown A.baumannii as the commonest organism. This is in accordance with other studies which have shown previous antibiotic therapy is the most important risk factor for developing MRSA infection (Wandirink et al., (1991)). This fact is well brought out from the present study, since most patients in this study, was on antibiotic therapy prior to ICU admission for various reasons and subsequently has grown MRSA.

Predominence of GNB is because in mechanically ventilated patients colinisation of respiratory tract occur with GNB and thus organisms are from the patient's own oropharyngeal and upper gastro - intestinal flora (Lumburt et al., (1989), Umberto, 1995, Bartlet, 1989). Tracheal colonisation without pneumonia clearly occurs in patients whose lungs are mechanically ventilated or who have undergone tracheostomy. If Pseudomonas is present in the tracheal aspirate culture of a patient with pneumonia it is very likely to be the causative organism. Moreover Pseudomonas species is known to colonize the trachea-bronchial tree without first appearing in the oropharyngeal secretions of intubated patients, presumably entering the lung via direct colonisation. This increases the incidence of Pseudomonas in mechanically ventilated individuals. This study has also shown polymicrobial etiology. But, considering $10^{6}$ $\mathrm{cfu} / \mathrm{ml}$ as the threshold except one, all other organisms did not show significant growth. Patients were enrolled as per the criteria. QETA and QBAL cultures were done in patients, both fitting and not fitting into clinical criteria to evaluate the counts in the 
presence and absence of clinical signs and symptoms.

Since most studies have shown a good sensitivity and specificity, taking $10^{6} \mathrm{cfu} / \mathrm{ml}$ and $10^{4} \mathrm{cfu} / \mathrm{ml}$ as the threshold for QETA and QBAL, the same threshold values were taken for the present study also. Also Gram smears of respiratory secretions (ETA \& BAL), were examined for the presence of $\geq$ 25 pus cells/10x, $\leq 10$ squamous epithelial cells/10x and ICO to access the quality of the sample.

\section{Evaluation of ETA Gram Smear with Clinical Criteria for the Diagnosis of VAP}

It is seen from this study that, $77 \%$ of patients in respiratory sample of the Gramstained smear being suggestive of pneumonia, may still not have clinical signs and symptoms, suggesting pneumonia. On the contrary, if the Gram-smear is not suggestive of VAP, it is unlikely that the patient $(67 \%)$ will have clinical signs and symptoms of pneumonia. So, a suggestive Gram-smear has to be always correlated with the clinical signs and symptoms for the diagnosis of VAP.

In this study, the sensitivity, specificity, PPV and NPV's of Gram-smear examination of ETA in cases with or without clinically diagnosed pneumonia was found to be $80 \%, 24.2 \%, 39 \%$ and $67 \%$ respectively. Therefore, Gram-smear examination of ETA has high sensitivity $(80 \%)$ but lower specificity $(62 \%)$. This is in accordance with other studies (Francois et al., 2(000)).

In this study, patients with Gram-smear suggestive of VAP with intracellular organism were found to be 11 out of 40.10 of the samples showing ICO also had significant growth in culture but only 4 of these were fitting into clinical criteria.
Evaluation of BAL Gram-Smear with Clinical Criteria for the Diagnosis of VAP

From this study it was seen that $67 \%$ of patients in the respiratory sample of the Grams-stain smear being suggestive of pneumonia, may still not have clinical signs and symptoms suggesting pneumonia. If the Gram-smear is not suggestive of VAP also, it is likely that many patients $(70 \%)$ may still have clinical signs and symptoms suggestive of pneumonia. So, both suggestive and non-suggestive Gram-smear has to be correlated with the clinical signs and symptoms for the diagnosis of VAP

The sensitivity, specificity, PPV and NPV of Gram smear are $41.6 \%, 23.08 \%, 33 \%$ and $30 \%$ respectively. So, Gram-smear of BALF lacks both sensitivity as well as specificity. This was contradictory to other studies which have shown high specificity (Pugin et al., (1991), Inglis et al., (1989)). Whereas Gram-smear examination of ETA obtained by bronchoscope had good sensitivity ( 24 of 27) but lacked specificity (34 false positives out of 57).

Also in this study, 5 of the smears revealed ICO out of a total of 15 smears which were suggestive of VAP. All the samples with ICO did show significant growth in culture but the clinical criteria were satisfied only in 2 patients. The percentage of cells containing intracellular bacteria in BAL has been proposed to diagnose VAP (Mauricio et al., (2000), Steven et al., (1997)).

\section{Evaluation of ETA Culture with Clinical Criteria for the Diagnosis of VAP}

From this study it is seen that $59 \%$ of patients culture results being suggestive of pneumonia, (significant growth $\geq$ $106 \mathrm{cfu} / \mathrm{ml}$ ) may still not have clinical signs and symptoms, suggesting pneumonia. On the contrary, if the culture results are not 
suggestive of VAP, it is unlikely that the patient (78\%) will have clinical signs and symptoms of pneumonia. So, a significant growth in culture has to be always correlated with the clinical signs and symptoms for the diagnosis of pneumonia. The sensitivity, specificity, PPV and NPV's of culture are $90 \%, \quad 21.2 \%, \quad 40.91 \%$ and $77.7 \%$ respectively. This is in par with other studies (Steven et al., (1997)).

From the study it is seen that QETA cultures for the diagnosis of VAP has greater sensitivity (90\%) and NPV (77\%). Even though the sensitivity is high, the specificity is lacking and so, the technique is hampered by false positive results. Hence, QETA cultures are best interpreted by taking into account the clinical criteria as well. Evaluation of BAL Culture with clinical criteria for the diagnosis of VAP: It is seen from this study that, $59 \%$ of patients culture results being suggestive of pneumonia, may still not have clinical signs and symptoms suggestive of VAP. If the culture is not suggestive of VAP also, it is likely that, many patients $(62.5 \%)$ may still have clinical signs and symptoms suggestive of pneumonia. So both significant as well as non-significant cultures have to be always correlated with the clinical signs and symptoms for the diagnosis of VAP

Table.1 Pattern of Bacterial Growth in ETA, BALF and Blood Culture

\begin{tabular}{|c|c|c|c|}
\hline \multirow[b]{2}{*}{$\begin{array}{l}\text { Bacterial culture } \\
\text { organsims }\end{array}$} & ETA & BAL & \multirow{2}{*}{$\begin{array}{l}\text { Blood } \\
\text { culture }\end{array}$} \\
\hline & $\begin{array}{l}\text { No.of cases in } \\
\text { which isolated }\end{array}$ & $\begin{array}{l}\text { No.of cases in } \\
\text { which isolated }\end{array}$ & \\
\hline $\begin{array}{l}\text { Pseudomonas aeruginosa } \\
(P . \text { aeruginosa) }\end{array}$ & $9(21.95 \%)$ & $3(13.6 \%)$ & $1(2.86 \%)$ \\
\hline Pseudomonas paucimobilis & $1(2.44 \%)$ & $0(0 \%)$ & $0(0 \%)$ \\
\hline $\begin{array}{l}\text { Acinetobacter } \\
\text { baumannii(A.baumannii) }\end{array}$ & $3(7.32 \%)$ & $5(22.7 \%)$ & $1(2.86 \%)$ \\
\hline $\begin{array}{l}\text { Klebsiella pneumoniae } \\
\text { (K.pneumoniae) }\end{array}$ & $3(7.32 \%)$ & $2(9 \%)$ & $0(0 \%)$ \\
\hline Staphylococcus aureus (S.aureus) & $3(7.32 \%)$ & $0(0 \%)$ & $6(17.14 \%)$ \\
\hline Streptococccus species & $3(7.32 \%)$ & $1(4.5 \%)$ & $0(0 \%)$ \\
\hline Citrobacter diversus (C.diversus) & $2(4.88 \%)$ & $0(0 \%)$ & $0(0 \%)$ \\
\hline $\begin{array}{l}\text { Hemophilus influenza } \\
\text { (H.influeza) }\end{array}$ & $1(2.44 \%)$ & $0(0 \%)$ & $0(0 \%)$ \\
\hline Coryneabacterium species & $3(7.32 \%)$ & $3(13.6 \%)$ & $0(0 \%)$ \\
\hline Escherichi coli $(E$ coli $)$ & $4(9.76 \%)$ & $3(13.6 \%)$ & $0(0 \%)$ \\
\hline Enterobacter species & $1(2.44 \%)$ & $0(0 \%)$ & $0(0 \%)$ \\
\hline Flavobacterium species & $1(2.44 \%)$ & $0(0 \%)$ & $0(0 \%)$ \\
\hline Proteus mirabilis(P.mirabilis) & $0(0 \%)$ & $1(4.5 \%)$ & $1(2.86 \%)$ \\
\hline No oraganism was grown & $7(17.07 \%)$ & $7(31.8 \%)$ & $26(74.29 \%)$ \\
\hline Total & 41 & 22 & 35 \\
\hline
\end{tabular}


Table.2 Comparison of ETA Gram Smear with Clinical Criteria

\begin{tabular}{|l|c|c|c|}
\hline \multirow{2}{*}{ Gramstain ETA } & \multicolumn{2}{|c|}{ Clinical criteria } & \multirow{2}{*}{ Total } \\
\cline { 2 - 3 } & Fitting & Not Fitting & 41 \\
\hline Suggestive & $16(39 \%)$ & $25(61 \%)$ & 12 \\
\hline Not suggestive & $4(33 \%)$ & $8(67 \%)$ & 53 \\
\hline Total & 20 & 33 & \\
\hline
\end{tabular}

Table.3 Comparison of ETA Gram Smear with Clinical Criteria

\begin{tabular}{|l|c|c|c|}
\hline \multirow{2}{*}{ BAL Gramstain } & \multicolumn{2}{|c|}{ Clinical Criteria } & \multirow{2}{*}{ Total } \\
\cline { 2 - 3 } & Fitting & Not Fitting & \\
\hline Suggestive & $5(33 \%)$ & $10(67 \%)$ & 15 \\
\hline Not suggestive & $7(70 \%)$ & $3(30 \%)$ & 10 \\
\hline Total & 12 & 13 & 25 \\
\hline
\end{tabular}

Table.4 Evaluation of ETA Culture with Clinical Criteria

\begin{tabular}{|l|c|c|c|}
\hline \multirow{2}{*}{ ETA Culture } & \multicolumn{2}{c|}{ Clinical criteria } & \multirow{2}{*}{ Total } \\
\cline { 2 - 3 } & Fitting & Not Fitting & 44 \\
\hline Suggestive & $18(41 \%)$ & $26(59 \%)$ & 9 \\
\hline Not suggestive & $2(22 \%)$ & $7(78 \%)$ & 53 \\
\hline Total & 20 & 33 & \\
\hline
\end{tabular}

Table.5 Evaluation of BAL Culture with Clinical Criteria

\begin{tabular}{|l|c|c|c|}
\hline \multirow{2}{*}{\multicolumn{1}{|c|}{ BAL Culture }} & \multicolumn{2}{c|}{ Clinical criteria } & \multirow{2}{*}{ Total } \\
\cline { 2 - 3 } & Fitting & Not Fitting & 17 \\
\hline Suggestive & $7(41 \%)$ & $10(59 \%)$ & 8 \\
\hline Not suggestive & $5(62.5 \%)$ & $3(37.5 \%)$ & 25 \\
\hline Total & 12 & 13 & \\
\hline
\end{tabular}


Table.6 Comparison of ETA Gram-stain with Culture

\begin{tabular}{|l|c|c|c|}
\hline \multirow{2}{*}{\multicolumn{1}{|c|}{ Gram stain }} & \multicolumn{2}{c|}{ Culture ETA } & \multirow{2}{*}{ Total } \\
\cline { 2 - 3 } & significant & Not significant & 41 \\
\hline Suggestive & $38(93 \%)$ & $3(7 \%)$ & 12 \\
\hline Not suggestive & $6(50 \%)$ & $6(50 \%)$ & 53 \\
\hline Total & 44 & 9 & 5 \\
\hline
\end{tabular}

Table.7 Comparison of BAL Gram-stain with Culture

\begin{tabular}{|l|c|c|c|}
\hline \multirow{2}{*}{\multicolumn{1}{|c|}{ Gram stain }} & \multicolumn{2}{c|}{ Culture BAL } & \multirow{2}{*}{ Total } \\
\cline { 2 - 3 } & significant & Not significant & \\
\hline Suggestive & $13(87 \%)$ & $2(13 \%)$ & 15 \\
\hline Not suggestive & $4(40 \%)$ & $6(60 \%)$ & 10 \\
\hline Total & 17 & 8 & 25 \\
\hline
\end{tabular}

\section{Percentage of patients with comorbid illness Fig -1}

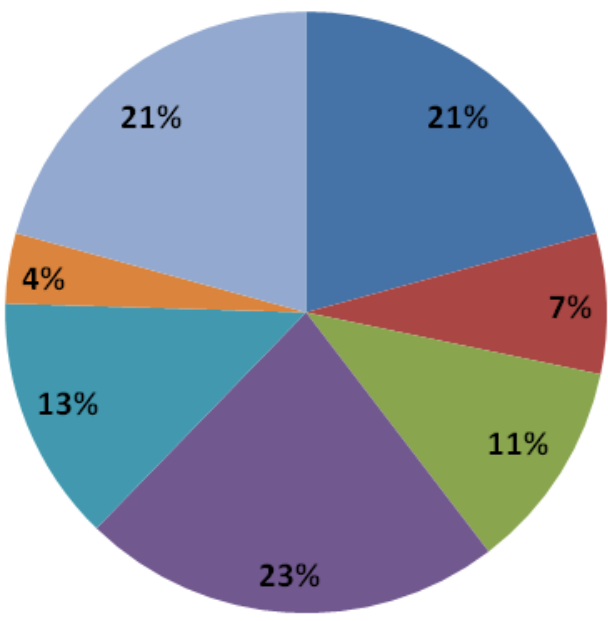

- cardiovascular

central nervous system

metabolic

resipiratory

Abdominal sepsis

Renal

Others 
Fig.2 Comparison of ETA Gram smear with Clinical Criteria

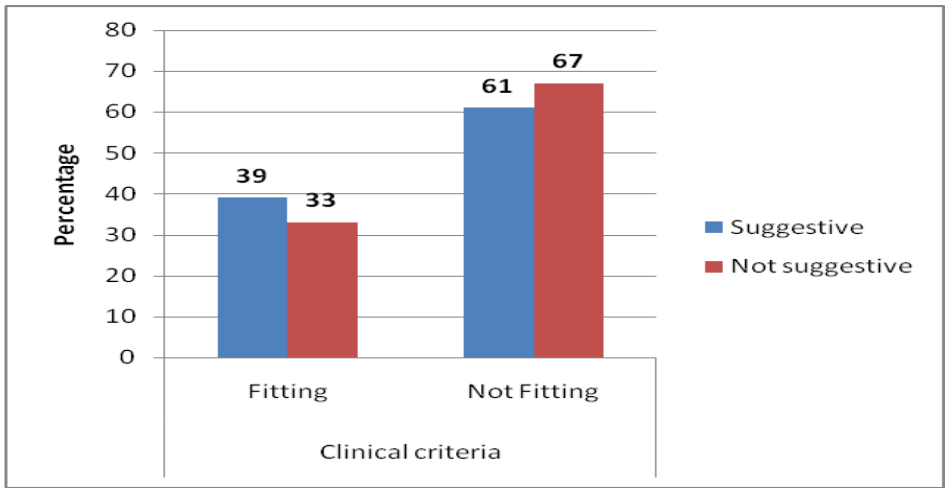

Fig.3 Comparison of BAL Gram stain with Clinical Criteria

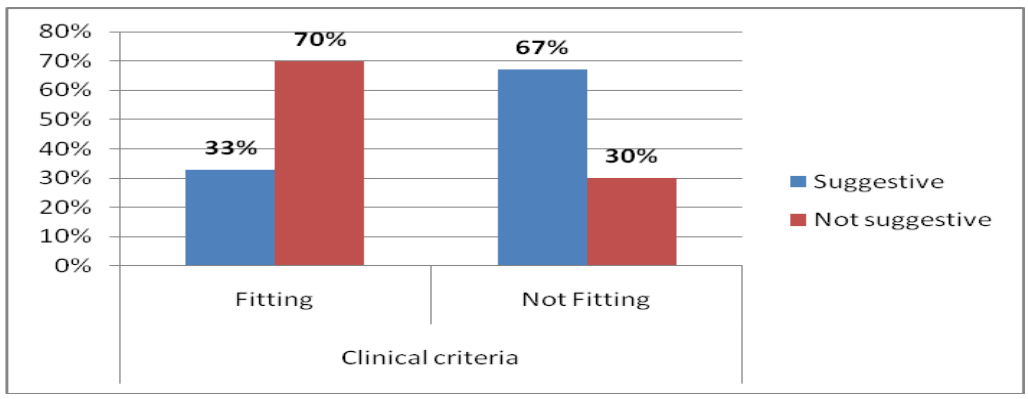

Fig.4 Evaluation of ETA Culture with Clinical Criteria

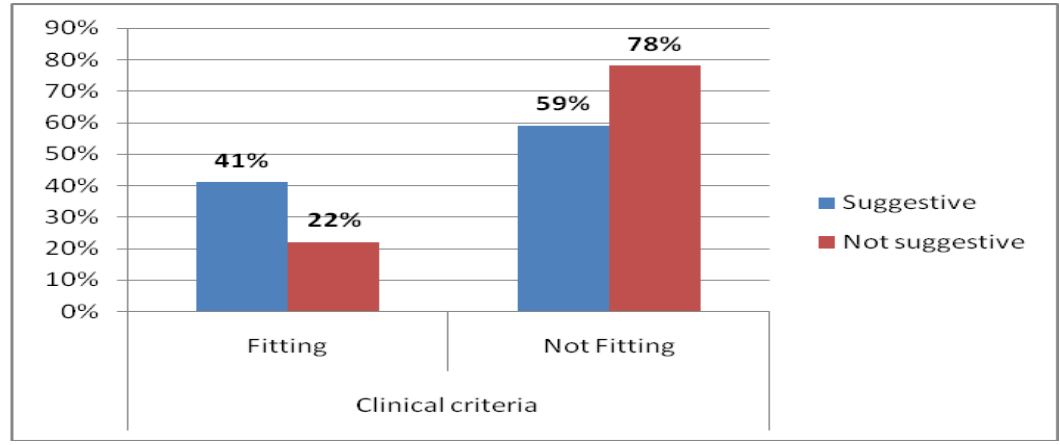

Fig.5 Evaluation of BAL culture with clinical criteria

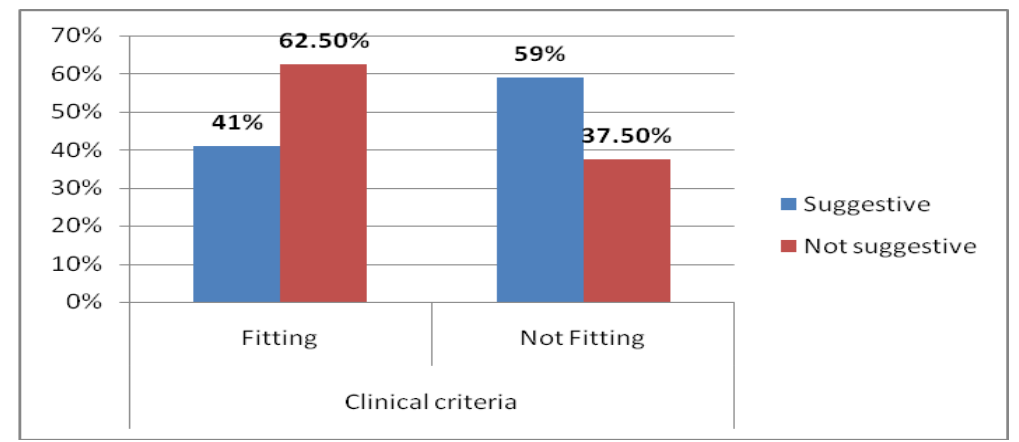


Fig.6 Comparison of ETA Gram smear with Clinical Criteria

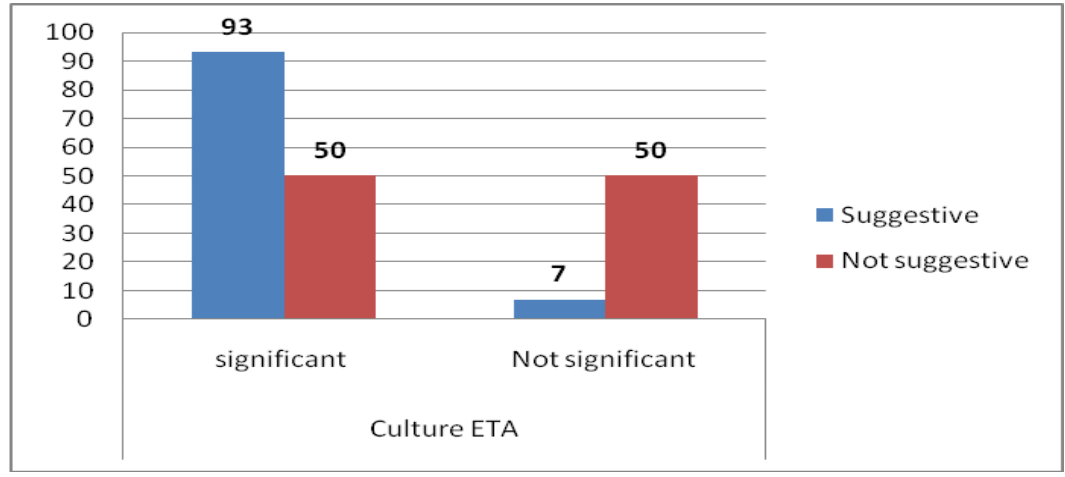

Fig.7 Comparison of BAL Gram-stain with Culture

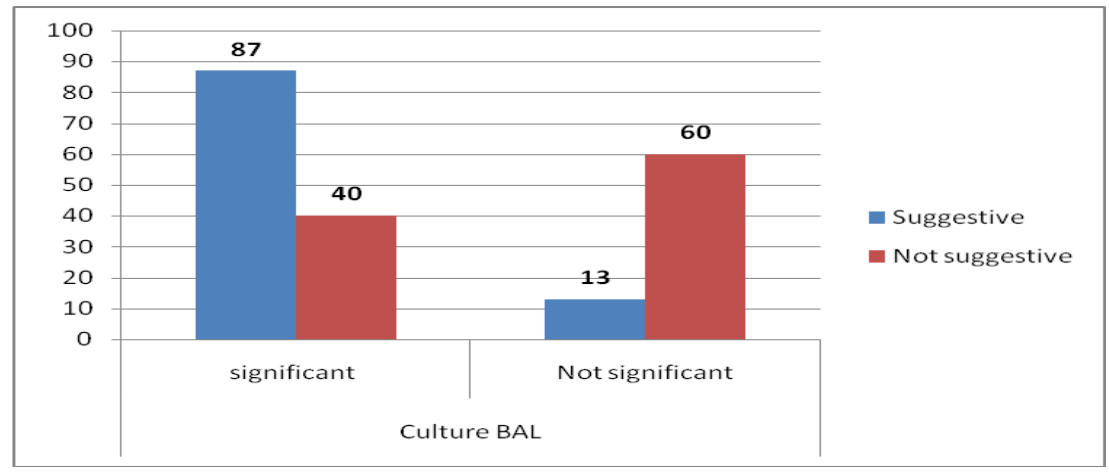

The sensitivity, specificity, PPV and NPV's for the culture are $58 \%, 23 \%, 41 \%$ and $37.5 \%$ respectively. This study revealed low sensitivity and specificity. This is in contrast to various other studies which have shown that QBAL cultures are superior and reliable in the diagnosis of VAP (Fagon et al., (1993), Glen, 2001). This may be because, the sample collection in this study was done from the 6th bronchoalveolar segment. Greater sensitivity and specificity can be probably be obtained if samples are collected from the lower bronchoscopic segments and possibly with a lesser dilution with the lavage fluid.

\section{Evaluation of ETA Gram-Smear with Culture for the Diagnosis of VAP}

In this study it was found that, if the Gramsmear is suggestive of VAP, majority of patients (92.6\%) also have significant growth suggestive of VAP, and if the Gramsmear is not suggestive of VAP, the possibilities of patients showing significant growth is equivocal. So, if the Gram-smear is not suggestive of pneumonia, the significant growth in culture may be correlated with clinical signs and symptoms of pneumonia. The sensitivity, specificity, PPV and NPV's of Gram-smear is $86 \%$, $66.67 \%, 92.6 \%$ and $50 \%$ respectively which is in par with other studies (Francois et al., (2000)).

\section{Evaluation of BAL Gram-Smear with Culture for the Diagnosis of VAP}

This study shows that, if the Gram-smear is suggestive of pneumonia, majority of patients $(86.6 \%)$ also show significant growth suggestive of pneumonia and if the 
Gram-smear is not suggestive of pneumonia, it is likely that many patients $(60 \%)$ may not also show significant growth suggestive of pneumonia. The sensitivity, specificity, PPV and NPV's of Gram-smear are $76.5 \%, 75 \%$, $86.7 \%$ and $60 \%$ respectively (Umberto, 1995).

Blood Culture: The growth of an organism in blood culture in the presence of clinical signs is always taken as the gold standard and an indicator for bacteremia in the presence of septic focus. This is more true in VAP, where the growth of the identical organism in blood culture as well as ETA or BAL carries a high diagnostic value. Therefore, blood cultures were done in 35 of the patients in this study at the time of obtaining sample for ETA or BAL. Out of 35 patients, 32 patients had QETA and blood culture done and in 16 patients QBAL and blood culture were done. Among the patients in whom blood culture and QETA were done, growth in blood culture was obtained in 7 patients. But the same organism in both ETA and blood culture were seen only in 4 patients. So, 3 out of the 7 patients who had bacteremia, the growth in blood culture was in variance with the growth in ETA. This reflects probably a skin contamination at the time of collecting blood or the non-specific nature of the ETA results.

Among the 16 patients in whom blood culture and QBAL were done, growth in blood culture was obtained in 4 patients of which 2 patients have grown the same organism both in BAL and blood culture. The other 2 patients who had bacteremia, the growth in blood culture was in variance with the growth in BAL. This shows that blood culture is not a very valuable technique because at most times there was no growth in culture. Similar studies conducted by others have also shown the same results
(Fagon et al., (1998), Meduri, 1993). This was probably because most patients have been administered antibiotics at the time of admission into ICU or from the start of their ICU stay. Collection of more than one sample of blood with optional time intervals may increase the sensitivity of blood culture as a diagnostic tool.

In conclusion, this study like other similar studies has shown the following.

Value of Gram-Smear Study: Gram-smear examination of ETA has high sensitivity but low specificity. Presence of ICO always correlated with the significant growth in culture. Therefore, the initial management of patients with suspected VAP, could be based on the carefully studied Gram-stain smear of ETA. Gram-smear of BALF lacks both sensitivity as well as specificity. Therefore this may not have much predictive value in the diagnosis of VAP. This is contrary to other studies.

Value of QETA and QBAL Cultures: QETA cultures for the diagnosis of VAP have greater sensitivity and negative predictive value taking $106 \mathrm{cfu} / \mathrm{ml}$ as the threshold value. Even though the sensitivity is high, the specificity is lacking as this technique is hampered by false positive results. Hence QETA cultures are best interpreted by taking into account the clinical criteria as well. But, since the specificity and PPV of QETA is almost the same as QBAL, QETA cultures can be reliably used for the diagnosis of VAP since this technique has got advantages like minimal risk, simplicity, less cost, less sideeffects and can be performed by bed side staff using standard therapeutic suction catheters.

QBAL cultures have shown low sensitivity and specificity probably because of the 
collection of sample from the 6th bronchoalveolar segment. Greater sensitivity can probably be obtained if samples are collected from the lower bronchoscopic segments and possibly with a lesser dilution with the lavage fluid. The use of a double lumen bronchoscopic catheter can probably increase the sensitivity and specificity of the procedures. Moreover bronchoscopic techniques are expensive, time consuming, give rise to complications, major risk of hypoxia in patients with ARDS and requires trained personnel.

Value of GSS with Cultures: Gram smear of ETA and BALF culture have high sensitivity and specificity. Therefore, the initial management of patients with suspected VAP could be based on Gram smear study.

Blood Cultures: Blood culture is not a very valuable technique because at most times there was no growth in culture. Multiple sampling at frequent intervals can achieve the yield in blood cultures but was not done in this study due to financial constraints.

\section{Acknowledgement}

My sincere gratitude to Dr. Vijayalakshmi kamat (HOD), DR. Arun kumar (Asst Prof) and Nurses of department of Anaesthesiology. We are extremely grateful to Dr. U.Krishna rao, Dept. of TB and Chest who performed broncoscopy procedures; the technicians of the department of microbiology for their support. Our sicere thanks to all the patients without whom this study would not have been possible.

\section{References}

American Thoracic society. 2005. Infectious Diseases society of America. Guidelines for the management of adult with hospital acquired, ventilator associated and Health care associated Pneumonia. Am. J. Respir. Crit. Care Med., 171: 388-416.

Bartlet, J.C., O Keefe, F.P., Tally, et al., 1986. Bacteriology of hospital acquired pneumonia Arch, Intern Med., 146: 868-871.

Bartletl, J.G. 1989. Invasive diagnostic technique in pulmonary infections in penngton JE respiratory infections. Diagnosis and management: New York, Raven pressive, 2: PP 52-68.

Baselshi, V. 1993. Microbiological diagnosis of ventilator - associated pneumonia. Infect. Dis. North Am., 7: 331-357.

Berton, D.C., Kalit, A.C., Teixeira, P.J. Quantitative versus quatitative cultures of respiratory secretions for clinical out.. in patients with ventilator associated pneumonia Cochrane Database syst. Rev.1 : CD006482.10.1002/ 14651858. CD 006482. Pub 2 ( Pub Med) ( Cross Ref)

Bonten, M.J., Weinstein, R.A. 1996. The role of colonization in the pathogenesis of nosocomial infections. Infect. Control Hosp. Epidemiol., 17: 193-200. 10. 2307/ 30142385 (Pub Med) (Cross Ref)

Brusselaers, N., Labeau, S., Vagelaers, D., Blot, S. 2013. Value of lower respiratory tract surveillance cultures to predict bacterial pathogens in Ventilator associated pneumonia: systematic review and diagnostic test occuracy meta- analysis. Intensive Care Med., 39: 365-375. 10.1007/s00134-012-2759 - x (Pub Med) (Cross Ref)

Camargo, L.F., De Marc, F.V., Barbas, C.S., et al., 2004. Ventilator associated pneumonia: comparison between quantitative and quantitative cultures 
of tracheal aspirates. Critical Care, 8: R422-30.

Collee, J.G., Miles, R.S., Watt, B. 2007. Tests for the identification of bacteria in: Colle JG, transfer AG, Marmion Craven, E., Chroneou, A. 2005. Nosocomial Pneumonia In: Mandel G, Bennette Dolin R, editors. Principles and Practice of Infectious Diseases. $7^{\text {th }}$ ed. London: Elsevier chruchill living stone, P. 3717-24.

El- Ebairy, M., Torres, A., Gonzalez, J., et al., 1993. Quantitative cultures of endotracheal aspirates for the diagnosis of ventilator associated pneumonia. Am. Rev. Respir. Dis., 148(6): 1552-7.

Fagon, J., Chastre, J., Hence, A. 1993. Evaluation of clinical judgment in the identification and treatment of nosocomial pneumonia in ventilated patients: Chest, 103: 547-553.

Fagon, J.Y., Chastre, J., Hence, A.J., et al., Detection of nosocomial lung infection in ventilated patients: Use of a protected specimen brush and quantitative culture techniques in 147 patients. Am. Rev. Respir. Dis., 138: 110-116.

Francois, Blot, Bruno paynard, Elisebath chachaty et al., 2000. Value of Gram stain examination of lower respiratory tract secretions for early diagnosis of nosocomial pneumonia: Am. J. Respi.r crit. care med., 162(5): 17311737.

Glen Mayhall, C. 2001. Ventilator associated pneumonia or not, Contemporary diagnosis: Emerg. Infect. Dis., Vol. 7, No. 2.

Grant, W., Waterer, M.B.B., Fracd, Richard, G., Wunderingk, M.D. 2001. Controversies in the diagnosis of ventilator- acquired pneumonia. Med. Clin. North America, Vol.85 Number. 6.
BP, Simmons A, editors. Mackie and McCarney: Practical Medical Microbiology $14^{\text {th }}$ ed. New Delhi: Churchill livingstone; 2007. P. 131-9.

Inglis, T.J., Millar, M.E., Jones, G., Robinson, D.A. 1989. Tracheal tube biofilm as a source of bacterial colonization of the lung: J. Clin. Microbial., 27: 2014-2018.

Lumbert, R.S., Vereen, L.E., George, R.B. 1989. Comparision of tracheal aspirates and Protected brush catheter specimens for identifying pathogenic bacteria in MV Patients: Am. J. Med. Sci., 297: 377-382.

Mastertpm, R.G., Gralloway, A., French, G., et al., 2008. Guidelines for the management of hospital acquired pneumonia in the UK: report of the working party on hospital acquired pneumonia of the British Society for Antimicrobial chemotherapy. $J$. Antimicrob. Chemother., 62: 5-34. 10.1093/jac/dkn162(Pub Med) (Cross Ref)

Mauricio Ruiz, Antoni Torres, Santiago Eurig, et al., 2000. Non-Invasive Versus Invasive Microbial investigation in VAP: Am. J. Respir. Git care Med., 162: 119-125.

Medford, A.R., Husain, S.A., Turki, H.M., Millar, A.B. 2009. Diagnosis of ventilator - associated pneumonia. $J$. Crit. Care, 24: 473. e 471-e 476.10.1016/j.jerc. 2008.06.012 (Pub Med) (Cross Ref)

Meduri, G.U. 1993. Diagnosis of VAP : Infect. Dis. Clin. North. Am., 7: 295329.

Meduri, G.U., Beals, D.H., Maijub, A.G., Baselski, V. 1991. Protected Bronchi - alveolar lavage - a new bronchoscopic technique to receive un contaminated distal airway secretions: Am. Rev. Respir. Dis., 855-864. 
Mozzamil Shafi, Syed Moied Ahmed, Managir Athar, et al., 2015. correlation between Tracheal Aspirate Culture and Bronchoalveolar Lavage culture for the diagnosis of Ventilator associated Pneumonia. Mt. J. Curr. Mirobiol. App. Sci., special Issue-1: 143-149.

Prekates, A., Nanas, S., et al., 1998. J. Infect. Dis., 30(1): 43-7.

Pugin, J., Auckenthaler, R., Mili, N., et al., 1991. Diagnosis of VAP by bacteriologic analysis of bronchoscopic and nonbronchoscopic "blind" BALF: Am. Rev. Respir. Dis., 143: 1121-1129.

Raoof, S., Baumann, M.H. 2014. Critical care Societis collaborative 2014. An official multi society statement: Ventilator associated events: the new definition. Crit. Care Med., 42: 228229.

Steven, H., Kirttand, M.D., David, E., Corley, M.D. et al., 1997. The diagnosis of VAP: Chest 112/2/1997 August

Umberto Meduri, G. 1995. FCCP: Diagnosis and differential diagnosis of VAP: Clin. Chest Med., Number, 1.

Wandirink, R.G., Rursell, G.B., Mezger, E., et al., 1991. The diagnostic utility of the ACB test in incubated Patients: Chest, 99: 84-88.

\section{How to cite this article:}

Sumana Prudhivi, Uma Sekhar, Anupama Jyoti Kindo, Mallika. 2016. Spectrum of Organisms and Correlation of Gram-Smear and Quantitative Cultures of Endotracheal Aspirates and Broncho Alveolar Lavage Fluid with Clinical Criteria for the Diagnosis of Ventilator Associated Pneumonia. Int.J.Curr.Microbiol.App.Sci. 5(5): 307-321. doi: http://dx.doi.org/10.20546/ijcmas.2016.505.033 